\title{
Órbitas esféricas de fótons ao redor de um buraco negro de Kerr
}

Photon spherical orbits around a Kerr black hole

\author{
Sérgio V. M. C. B. Xavier ${ }^{1}$, Luiz C. S. Leite ${ }^{1,2}$, Carolina L. Benone $\sqrt[*]{\square}$, Carlos A. R. Herdeiro ${ }^{4}$, \\ Luís C. B. Crispino1@ \\ ${ }^{1}$ Universidade Federal do Pará, Faculdade de Física, Belém, PA, Brasil. \\ ${ }^{2}$ Instituto Federal do Pará, Campus Altamira, Altamira, PA, Brasil. \\ ${ }^{3}$ Universidade Federal do Pará, Campus Universitário Salinópolis, Salinópolis, PA, Brasil. \\ ${ }^{4}$ Universidade de Aveiro, Centro de Investigação e Desenvolvimento em Matemática e Aplicações (CIDMA), Departamento de \\ Matemática, Aveiro, Portugal.
}

Recebido em 05 de maio de 2020. Revisado em 24 de junho de 2020. Aceito em 28 de junho de 2020.

\begin{abstract}
Buracos negros são regiões aprisionadas do espaço-tempo. A sua inexorável atração gravitacional determina que nada - nem mesmo a luz - pode escapar desta região. Logo, um buraco negro não pode ser observado diretamente. Tem de ser estudado pelo modo como influencia o movimento das partículas de matéria e os raios de luz ao seu redor. Estas trajetórias são determinadas (em uma certa aproximação) pelas geodésicas da geometria do buraco negro. Em particular, geodésicas nulas - aquelas que raios luminosos seguem - possuem considerável interesse astrofísico: representam como a radiação emitida ao redor do buraco negro, ou proveniente de fontes longínquas, é distorcida pelo buraco negro. Neste trabalho apresentamos as equações de movimento de raios de luz na geometria de Kerr, que descreve um buraco negro com rotação, utilizando a teoria de Hamilton-Jacobi. Em seguida, analisamos as condições de existência das órbitas esféricas de fótons ao redor do buraco negro. Estas órbitas, representando fortes encurvamentos da luz, são a chave para determinar a aparência ótica do buraco negro. Por fim, por meio de métodos computacionais, apresentamos graficamente algumas destas trajetórias. Palavras-chave: Relatividade Geral, Buracos Negros, Órbitas de Fótons.
\end{abstract}

Black holes are trapped spacetime regions. Their inexorable gravitational attraction determines that nothing not even light - may escape from this region. Thus, a black hole cannot be directly observed. It must be studied by the way it influences the motion of matter particles and light rays in its neighborhood. These trajectories are determined (within a certain approximation) by the black hole geometry's geodesics. In particular, null geodesics - those followed by light rays - possess considerable astrophysical interest: they represent how the radiation emitted around the black hole, or incoming from far away sources, is distorted by the black hole. In this work we present the equations of motion of light rays in the Kerr geometry, that describes a rotating black hole, making use of Hamilton-Jacobi theory. Subsequently, we analyze the conditions for the existence of spherical photon orbits around the black hole. These orbits, representing strong light bending, are the key to determine the optical appearance of the black hole. Finally, using computational methods, we exhibit plots illustrating some of these trajectories.

Keywords: General Relativity, Black Holes, Photon Orbits.

\section{Introdução}

Em 1915, o físico suíço-germano-norte americano Albert Einstein apresentou uma maneira geometrizada de entender a interação gravitacional, conhecida como Teoria da Relatividade Geral (TRG). Esta teoria descreve a gravidade como uma manifestação na geometria do espaço-tempo, causada pela presença de matéria e energia.

As equações que governam esta relação entre matéria e geometria levaram a predições surpreendentes, como a

*Endereço de correspondência: benone@ufpa.br possível existência de objetos suficientemente compactos, tais que exerceriam uma atração gravitacional tão intensa ao seu redor que possuam uma região de não-retorno (chamada de horizonte de eventos), de onde nem mesmo a luz seria capaz de escapar. Esses objetos são denominados buracos negros (BNs) [1].

Um século após a obtenção da primeira solução teórica que descreve um BN [2,3], existem evidências observacionais convincentes e variadas da sua realidade astrofísica [4]. Uma evidência indireta da existência de BNs foi obtida em 2015, por meio da detecção de ondas gravitacionais pelo Laser Interferometer Gravitational-Wave Observatory (LIGO) [5]. Este evento foi interpretado 
como tendo sido gerado pela coalescência de dois BNs, um com cerca de 36 massas solares e outro com 29 massas solares aproximadamente. Desde então, a colaboração LIGO-Virgo já reportou diversos eventos interpretados como colisões de BNs, colisões de estrelas de nêutrons e, possivelmente, colisões de estrelas de nêutrons com BNs.

Os BNs observados pela colaboração LIGO-Virgo têm massas que variam de algumas unidades, a algumas dezenas de unidades da massa do Sol. Mas existe uma classe diferente de BNs, que os astrônomos acreditam existir no núcleo da maioria das galáxias: BNs supermassivos, com massas variando de centenas de milhares a dezenas de bilhões de massas do Sol. Em todos os casos, esperase que, genericamente, os BNs possuam rotação 6 [9]. Nestes casos, a região externa destes objetos é descrita pela solução de Kerr, de acordo com a TRG. Obtida pelo matemático neozelandês Roy Patrick Kerr em 1963 [10, esta solução descreve um espaço-tempo estacionário e com simetria axial.

Contudo, nos deparamos com um impasse: se BNs parecem ser objetos abundantes no Universo e, por outro lado, nada escapa do interior do horizonte de eventos, como podemos observá-los? Uma das maneiras é analisar a influência do campo gravitacional no movimento de corpos e da luz ao redor de um BN. Este tipo de análise é tão importante que um dos primeiros testes da TRG foi realizado medindo-se a deflexão sofrida por raios luminosos ao atravessarem o campo gravitacional solar durante um eclipse $[11-13]$.

O estudo das trajetórias seguidas por partículas sujeitas apenas à interação gravitacional se prova uma importante fonte de informações acerca da geometria do espaço-tempo. Essas trajetórias no espaço-tempo são denominadas de geodésicas. Em particular, o estudo de geodésicas nula: $\mathfrak{1}^{1}$ ajuda a definir, por exemplo, a seção de choque de absorção (a altas frequências), isto é, a área associada ao conjunto de trajetórias assintóticas, que define a região em que partículas são absorvidas pelo $\mathrm{BN}^{2}[14 \sqrt{16}]$.

A seção de choque de absorção está relacionada com órbitas circulares de fótons, denominadas de anéis de luz (AL). Este encurvamento intenso da luz possui características fenomenológicas únicas, e estudos indicam que o sinal de ondas gravitacionais pós-colisão de BNs depende desses AL 17, 18]. Em particular, se existirem mecanismos que proíbam a existência de estrelas ultracompactas 19], então observações de AL podem ser consideradas evidências de detecção de BNs [20].

Neste sentido, órbitas de raio constante são importantes também por auxiliarem na determinação da sombra ${ }^{3}$

${ }^{1}$ Como a assinatura da métrica usada neste trabalho é $(+,-,-,-)$, a convenção de sinal adotada é: partículas massivas seguem trajetórias do tipo tempo, definidas por $d s^{2}>0$; e partículas não massivas seguem trajetórias nulas, definidas por $d s^{2}=0$.

2Entende-se por 'absorvidas pelo buraco negro' o fato de partículas ficarem aprisionadas no interior do horizonte de eventos.

${ }^{3}$ Imagine que entre o observador e uma fonte luminosa (por exemplo, estrelas) existe um BN interposto. Caso o observador tentasse tirar de BNs 21,22]. Recentemente, a colaboração internacional denominada Event Horizon Telescope (EHT) revelou, em uma série de seis artigos, ter obtido sucesso na observação da sombra do BN supermassivo localizado no núcleo da galáxia Messier 87 [23 29]. Para isso, esse projeto utilizou uma técnica chamada de Very-Long-Baseline Interferometry (VLBI), que consiste em sincronizar os dados de uma rede de radiotelescópios espalhados ao longo de quatro continentes do planeta, para obter uma resolução equivalente a de um telescópio do tamanho do planeta Terra.

A sombra de um BN de Kerr está estritamente associada com uma classe de órbitas nulas chamadas de órbitas esféricas de fótons ( $\mathrm{OEF})$. Podemos entender as $\mathrm{OEF}$ como uma generalização fora do plano equatorial dos AL. São as OEF que determinam o formato da sombra de um BN. Em casos de modelos mais gerais de BNs as OEF são generalizadas para as órbitas fundamentais de fótons [30], que estão confinadas entre um raio máximo e um raio mínimo, mas que não são necessariamente esféricas 4 em nenhum sistema de coordenadas.

A detecção de ondas gravitacionais e a captura da primeira imagem de um buraco negro inauguram o período de testes da gravitação em campo forte, nos levando mais perto da formulação de uma teoria quântica da gravitação. Neste contexto, a análise de OEF em espaços-tempos de BNs com rotação mostra-se uma importante ferramenta para estudar a física destes objetos. Deste modo, realizamos neste trabalho uma análise dos principais aspectos dessas trajetórias. Com isto, esperamos contribuir com uma referência pedagógica para aqueles interessados em entender a formação da sombra de buracos negros, além de servir como um possível material de apoio para cursos como RG ou Mecânica Clássica.

O restante deste artigo está dividido como se segue: Na seção II revisitamos brevemente as principais características do espaço-tempo de um BN em rotação. Na seção III, revisamos a teoria de Hamilton-Jacobi, importante ferramenta necessária para encontrar as equações de movimento de partículas teste. Na seção IV, aplicamos esta teoria no contexto da TRG para obtermos as geodésicas do espaço-tempo de Kerr. Uma vez obtidas estas trajetórias, realizamos uma discussão, na seção $\mathrm{V}$, sobre as equações que devem ser satisfeitas para que existam órbitas esféricas nesta geometria, bem como plotamos alguns exemplos de OEF. Por fim, nossas conclusões são apresentadas na seção VI.

Assume-se que o leitor tenha conhecimentos prévios de TRG. Caso contrário, recomendamos consultar as referências 3133 .

uma foto deste cenário, a região escura no retrato identificaria a região em seu céu local que não recebe luz, pois esta teria sido absorvida pelo BN. Essa região escura na foto seria a sombra do BN.

${ }^{4}$ Ver comentário ao final da seção V. 


\section{Buraco Negro de Kerr}

O BN de Kerr é descrito por dois parâmetros [34]: sua massa $M$ e seu momento angular $J$. Utilizamos como parâmetro de rotação o momento angular por unidade de massa, $a \equiv J / c M$. 5 Em coordenadas de BoyerLindquist 35 o elemento de linha de Kerr tem a forma:

$$
\begin{aligned}
d s^{2}= & c^{2}\left(1-\frac{2 m r}{\rho^{2}}\right) d t^{2}+\frac{4 m a c r \sin ^{2} \theta}{\rho^{2}} d t d \phi-\frac{\rho^{2}}{\Delta} d r^{2} \\
& -\rho^{2} d \theta^{2}-\left(r^{2}+a^{2}+\frac{2 m r a^{2} \sin ^{2} \theta}{\rho^{2}}\right) \sin ^{2} \theta d \phi^{2}
\end{aligned}
$$

com $\Delta \equiv r^{2}-2 m r+a^{2}$ e $\rho^{2} \equiv r^{2}+a^{2} \cos ^{2} \theta$. Além disso, definimos como parâmetro de massa $m \equiv G M / c^{2}$, onde $G$ é a constante newtoniana da gravitação. Apesar de $m$ não ter unidade de massa, $m$ deve ser interpretada como a massa do BN medida em unidades de distância 36.

Trabalharemos com o caso em que a condição $a^{2}<m^{2}$ é satisfeita. Este vínculo corresponde a um BN para o qual o horizonte de eventos está localizado em $r_{+}=$ $m+\sqrt{m^{2}-a^{2}}$. O caso $a^{2}>m^{2}$ representa um objeto chamado singularidade nua. Neste contexto, singularidade refere-se a um ponto, ou conjunto de pontos, no espaço-tempo onde a curvatura é infinita e, como tal, a TRG deixa de ser válida. Uma singularidade nua significa que a singularidade é visível para observadores, o que coloca sérias dificuldades à formulação do problema de valor inicial 37$]^{6}$ A existência de singularidades nuas é tão inconveniente que o físico Roger Penrose propôs em 1969 a Censura Cósmica, que afirma que qualquer singularidade deve estar escondida no interior de um horizonte de eventos 36. O caso $a^{2}=m^{2}$ corresponde a um $\mathrm{BN}$ com rotação máxima, também chamado de $\mathrm{BN}$ de Kerr extremo.

A métrica de Kerr apresenta uma região de não estaticidade, denominada ergoregião. Nesta região não é possível a existência de observadores parados em relação a referenciais estáticos no infinito. A ergoregião se localiza na vizinhança do horizonte de eventos e é consequência da rotação desse horizonte. Qualquer observador nesta região é obrigado a se mover no mesmo sentido de rotação do BN 7]. Uma maneira de encontrá-la, por exemplo, é estudando as condições que a trajetória de um observador estático deve satisfazer. Este observador estático não possui variação nas coordenadas espaciais (i.e. $d r=d \theta=d \phi=0$ ). Além disso, a exigência que esta

\footnotetext{
${ }^{5}$ Definimos aqui que o sentido positivo de rotação é o sentido anti-horário.

${ }^{6}$ Para que uma teoria física tenha a capacidade de fazer previsões, ela deve possuir uma formulação do problema de valor inicial bem posta. Isto significa que a partir de um conjunto de dados iniciais de um sistema, é possível descrever a evolução temporal deste sistema em todo o espaço-tempo. Porém, quando singularidades nuas estão presentes, os dados iniciais não são suficientes para descrever o que ocorre quando o sistema alcança a singularidade (ou sua vizinhança), isto é, a habilidade de prever o futuro deste sistema é perdida nesta região do espaço-tempo.
}

trajetória represente uma partícula causal (isto é, uma partícula de matéria ou um fóton) é traduzida exigindo que

$$
\left.d s^{2}\right|_{\text {estático }}=c^{2}\left(1-\frac{2 m r}{\rho^{2}}\right) d t^{2} \geq 0 .
$$

É possível mostrar que essa condição não é satisfeita no intervalo definido por

$$
r_{+}<r<r_{E} \equiv m+\sqrt{m^{2}-a^{2} \cos ^{2} \theta},
$$

que determina a ergoregião, onde $r_{E}$ localiza o seu limite exterior.

A ergoregião é uma manifestação do efeito de arrasto de referenciais inerciais, que surge como um novo aspecto da gravitação relativística, ausente na gravitação Newtoniana. Na gravitação relativística, o momento linear e o momento angular também gravitam, originando novos efeitos, ao contrário da gravitação Newtoniana, onde apenas a posição das massas interessa, e não o seu estado de movimento em um certo instante. Neste sentido, o estudo de geodésicas no contexto de um BN de Kerr é mais complicado do que no BN de Schwarzschild ou de Reissner-Nordström [38], que não têm rotação.

Ao considerar o movimento de partículas somente sob a ação da gravidade na métrica de Kerr, as quantidades conservadas no movimento geodésico desempenham um papel fundamental. A energia da partícula, bem como o seu momento angular relativo ao eixo de simetria são quantidades conservadas, como consequência das simetrias da métrica de Kerr e do teorema de Noether [39]. Logo, as geodésicas em um espaço-tempo estacionário e com simetria axial permitem duas integrais de movimento. Além disso, o módulo do quadrimomento dessa partícula também é uma quantidade conservada, sendo determinado pela massa de repouso da partícula. Contudo, tais grandezas conservadas não são suficientes para tornar o problema integrável, no sentido de Liouville [39]. Para isso, é necessário ter tantas quantidades conservadas como graus de liberdade. No movimento no espaço-tempo há quatro graus de liberdade e identificamos apenas três constantes de movimento.

Surge então uma característica peculiar da métrica de Kerr. Existe uma quarta constante de movimento, descoberta em 1968 pelo físico australiano Brandon Carter 40]. A existência dessa quarta constante de movimento está vinculada ao fato de o espaço-tempo de Kerr ter características geométricas especiais: É uma métrica do tipo D de acordo com a classificação de Petrov 7 Para espaçostempos do tipo D existem alguns teoremas que regem o movimento geodésico 41]. No seu trabalho original, Carter demonstrou que existe separação de variáveis para

\footnotetext{
${ }^{7}$ Para o caso de vácuo, a classificação de Petrov é uma maneira algébrica de caracterizar um campo gravitacional a partir do tensor de curvatura de Riemann. Ela se baseia na definição de uma equação de autovalor para o tensor de curvatura. Dependendo da degenerescência dos seus autovalores, o espaço-tempo pode ser classificado como um dos quatro tipos de Petrov. Para mais detalhes, sugerimos consultar a referência 41.
} 
a equação de Hamilton-Jacobi na métrica de Kerr 40]. Esta separação permite a identificação da quarta constante do movimento, chamada de constante de Carter. A sua existência resulta de uma simetria escondida, por oposição às simetrias manifestas que originam a conservação da energia e do momento angular das geodésicas. Neste trabalho, seguiremos o método de Carter para analisar geodésicas no espaço-tempo de Kerr.

\section{Teoria de Hamilton-Jacobi}

Nesta seção realizamos uma breve revisão acerca da teoria de Hamilton-Jacobi. Esta teoria é um método que consiste em encontrar uma transformação canônica tal que a nova hamiltoniana seja identicamente nula. Esta transformação corresponde a encontrar um referencial comóvel com a partícula no espaço de fase. Isto simplifica o processo de obtenção das equações de movimento 39 .

Conhecendo a transformação canônica que leve das coordenadas $\left(q_{i}, p_{i}\right)$ para novas coordenadas $\left(Q_{i}, P_{i}\right)^{8}$ e assumindo que $K\left(Q_{i}, P_{i}\right)$ seja a hamiltoniana transformada, as equações de Hamilton são:

$$
\begin{aligned}
& \dot{Q}_{i}=\frac{\partial K}{\partial P_{i}}=0 \quad \Rightarrow Q_{i}=\beta_{i}, \\
& \dot{P}_{i}=-\frac{\partial K}{\partial Q_{i}}=0 \quad \Rightarrow P_{i}=\alpha_{i},
\end{aligned}
$$

onde $\left(\beta_{i}, \alpha_{i}\right)$ são constantes e $i=1,2, \cdots, n$. O ponto sobre as variáveis representa uma derivada em relação ao parâmetro afim.

Escrevendo as coordenadas originais em termos das coordenadas transformadas, isto é, considerando a transformação canônica inversa, obtemos:

$$
\begin{aligned}
& q_{i}=q_{i}\left(Q_{i}, P_{i}, t\right) \quad \Rightarrow \quad q_{i}(t)=q_{i}\left(\beta_{i}, \alpha_{i}, t\right) \\
& p_{i}=p_{i}\left(Q_{i}, P_{i}, t\right) \quad \Rightarrow \quad p_{i}(t)=p_{i}\left(\beta_{i}, \alpha_{i}, t\right)
\end{aligned}
$$

Estas equações fornecem as coordenadas e os momentos generalizados em função do tempo e de $2 n$ constantes arbitrárias que podem ser definidas a partir das condições iniciais. Uma transformação canônica desse tipo torna triviais as equações de Hamilton para $K(Q, P, t)$, isto é, com soluções triviais, permitindo obter as soluções das equações de Hamilton para as variáveis originais por meio de uma transformada inversa.

Para obtermos uma transformação canônica deve existir uma quantidade que relacione as coordenadas antigas $\left(q_{i}, p_{i}\right)$ com as coordenadas novas $\left(Q_{i}, P_{i}\right)$. O objeto matemático que cumpre esta função é a função geradora 39. A partir da escolha da função geradora obtemos automaticamente uma transformação canônica. Neste contexto, podemos nos perguntar: que condição a função que gera

\footnotetext{
$8 q$ é uma coordenada generalizada e $p$ o momento canonicamente conjugado a esta coordenada. $Q$ e $P$ possuem, respectivamente, o mesmo significado que $(q, p)$, porém no novo sistema de coordenadas.
}

essa transformação deve satisfazer para que a hamiltoniana transformada seja nula? Para responder esta pergunta, consideremos uma função geradora do tipo $S\left(q_{i}, P_{i}, t\right)$, em que as equações de transformação são dadas por

$$
p_{i}=\frac{\partial S}{\partial q_{i}}, \quad Q_{i}=\frac{\partial S}{\partial P_{i}} .
$$

Deste modo, a hamiltoniana transformada é 39

$$
K\left(Q_{i}, P_{i}, t\right)=H\left(q_{i}, p_{i}, t\right)+\frac{\partial S}{\partial t} .
$$

Para que se tenha $K=0, S$ deve satisfazer a seguinte equação

$$
H\left(q_{i}, p_{i}, t\right)+\frac{\partial S}{\partial t}=0
$$

onde, usando as relações (8), obtemos:

$$
H\left(q_{i}, \frac{\partial S}{\partial q_{i}}, t\right)+\frac{\partial S}{\partial t}=0 .
$$

A condição 11 recebe o nome de equação de HamiltonJacobi. A função geradora $S$ deve satisfazer a equação de Hamilton-Jacobi para que $K=0$. Resolvendo esta equação para a função geradora, obtemos automaticamente uma transformação canônica com a propriedade de anular a nova hamiltoniana, e podemos encontrar as equações de movimento.

\section{Equação de Hamilton-Jacobi no espaço-tempo de Kerr}

Na TRG, a hamiltoniana é dada por 41]

$$
H\left(x^{\mu}, p_{\mu}, \lambda\right)=\frac{1}{2} g^{\mu \nu} p_{\mu} p_{\nu},
$$

onde $\lambda$ é um parâmetro afim, assumindo valores diferentes dependendo se a partícula estudada possui massa ou não. Neste contexto, a equação de Hamilton-Jacobi pode ser escrita como [42]:

$$
\frac{1}{2} g^{\mu \nu} \frac{\partial S}{\partial x^{\mu}} \frac{\partial S}{\partial x^{\nu}}=-\frac{\partial S}{\partial \lambda} .
$$

No espaço-tempo de Kerr, a hamiltoniana não possui dependência explícita em $t, \phi$ e $\lambda$. Portanto, a função $S$ pode ser expressa como 40

$$
S\left(x^{\mu} ; \alpha_{\mu} ; \lambda\right)=p_{t} t+p_{\phi} \phi+\gamma \lambda+S_{r}(r)+S_{\theta}(\theta),
$$

onde $p_{t} \equiv E$ e $p_{\phi} \equiv-L$ são os momentos canonicamente conjugados às coordenadas $t \mathrm{e} \phi$, respectivamente. Além disso,

$$
\frac{\partial S}{\partial \lambda}=\gamma=-H=-\frac{\epsilon^{2}}{2},
$$

onde, para partículas massivas, $\epsilon=1$ e $E$ e $L$ representam a energia e o momento angular azimutal por unidade de 
massa, e, para partículas não massivas, $\epsilon=0$ e $E$ e $L$ representam a energia e o momento angular azimutal.

Substituindo as quantidades conservadas ao longo do movimento geodésico, podemos reescrever a equação (14) como

$$
S\left(x^{\mu} ; \alpha_{\mu} ; \lambda\right)=-\frac{\epsilon^{2}}{2} \lambda+E t-L \phi+S_{r}(r)+S_{\theta}(\theta) .
$$

Substituindo a equação (16) na equação de HamiltonJacobi, após algumas manipulações algébricas, encontramos o seguinte resultado:

$$
\frac{\mathcal{B}^{2} / c^{2}-\Delta^{2} p_{r}^{2}-\epsilon^{2} r^{2} \Delta}{\Delta}=p_{\theta}^{2}+\mathcal{D}^{2}+\epsilon^{2} a^{2} \cos ^{2} \theta,
$$

onde

$$
\begin{aligned}
p_{r} & =\frac{\partial S}{\partial r}, \quad p_{\theta}=\frac{\partial S}{\partial \theta}, \\
\mathcal{B}^{2} & \equiv\left[E\left(r^{2}+a^{2}\right)-a L c\right]^{2}, \\
\mathcal{D}^{2} & \equiv\left(\frac{a E \sin \theta}{c}-\frac{L}{\sin \theta}\right)^{2} .
\end{aligned}
$$

Note que o lado esquerdo da equação 17 é função apenas da coordenada $r$, enquanto que o lado direito depende exclusivamente da coordenada angular $\theta$. Esta igualdade só é válida se ambos os lados forem iguais a uma constante

$$
f_{r}(r)=f_{\theta}(\theta)=\mathcal{K} .
$$

Define-se, no espaço-tempo de Kerr, a constante de Carter 40] $Q$ como:

$$
Q \equiv \mathcal{K}-\left(L-\frac{a E}{c}\right)^{2}
$$

Trabalhando separadamente com cada lado da equação (17), encontramos as equações de movimento para as coordenadas $r$ e $\theta$. As equações para $t$ e $\phi$ são encontradas usando o fato de que $p_{\mu}=\partial_{\mu} S$.

Deste modo, no espaço-tempo de Kerr, as equações de movimento na forma diferencial são:

$$
\begin{aligned}
\rho^{2} \dot{r} & = \pm \sqrt{R}, \\
\rho^{2} \dot{\theta} & = \pm \sqrt{\Theta}, \\
\rho^{2} \dot{t} & =\frac{E}{c^{2} \Delta}\left[\left(r^{2}+a^{2}\right)^{2}-a^{2} \Delta \sin ^{2} \theta\right]-\frac{2 m a r}{c \Delta} L, \\
\rho^{2} \dot{\phi} & =\frac{2 m a r E}{c \Delta}+L \frac{\left(\Delta-a^{2} \sin ^{2} \theta\right)}{\Delta \sin ^{2} \theta},
\end{aligned}
$$

com

$$
\begin{aligned}
R & \equiv \frac{\mathcal{B}^{2}}{c^{2}}-\Delta\left[Q+(L-a E / c)^{2}+\epsilon^{2} r^{2}\right], \\
\Theta & \equiv Q-\cos ^{2} \theta\left[a^{2}\left(\epsilon^{2}-\frac{E^{2}}{c^{2}}\right)+\frac{L^{2}}{\sin ^{2} \theta}\right] .
\end{aligned}
$$

Da equação (24) podemos perceber que a nulidade da constante de Carter está relacionada com trajetórias no plano equatorial $(\theta=\pi / 2)$.

\section{5. Órbitas esféricas de fótons}

Como definido anteriormente, para fótons teremos $\epsilon^{2}=$ 0 . Ademais, podemos reduzir o número de parâmetros $(E, L, Q)$ definindo os parâmetros de impacto:

$$
\begin{aligned}
& \Phi \equiv \frac{L}{E} c, \\
& \eta \equiv \frac{Q}{E^{2}} c^{2} .
\end{aligned}
$$

\subsection{Movimento latitudinal}

A equação (24), após a divisão por $E^{2}$ e a utilização das Eqs. 29) e (30), torna-se:

$\left(\frac{\rho^{2} c}{E}\right)^{2} \dot{\theta}^{2}=\eta+(\Phi-a)^{2}-(a \sin \theta-\Phi \operatorname{cossec} \theta)^{2}=\Theta(\theta)$. (31)

Note que para $\Theta \geq 0$ devemos ter

$$
\begin{aligned}
& \eta+(\Phi-a)^{2}>0, \\
& \eta+(\Phi-a)^{2} \geq(a \sin \theta-\Phi \operatorname{cossec} \theta)^{2} .
\end{aligned}
$$

Podemos escrever a equação (31) de uma maneira mais conveniente fazendo a transformação:

$$
u=\cos \theta .
$$

Assim, obtemos:

$$
\left(\frac{\rho^{2} c}{E}\right)^{2} \dot{u}^{2}=\eta-\left(\Phi^{2}+\eta-a^{2}\right) u^{2}-a^{2} u^{4}=\Theta_{u}(u) .
$$

Os valores extremos da função $u$ equivalem aos seguintes resultados correspondentes para a função $\Theta_{u}$ :

$$
\begin{aligned}
& u=-1 \Leftrightarrow(\theta=\pi) \Rightarrow \Theta_{u}=-\Phi^{2}, \\
& u=0 \Leftrightarrow\left(\theta=\frac{\pi}{2}\right) \Rightarrow \Theta_{u}=\eta, \\
& u=1 \Leftrightarrow(\theta=0) \Rightarrow \Theta_{u}=-\Phi^{2} .
\end{aligned}
$$

Assim, o intervalo permitido do movimento latitudinal pode ser restringido. Para $\Phi \neq 0$, os fótons não podem alcançar os polos $(\theta=0, \pi)$, uma vez que neste local a função $\Theta_{u}$ assume valores negativos, o que é incompatível com a equação (35). Apenas quando $\Phi=0$ teremos $\Theta_{u}=0$ nos polos, o que é permitido, haja vista que $\Theta_{u} \geq 0$. Então, a única maneira de fótons alcançarem os polos é quando o momento angular azimutal destes fótons for nulo [ver equação [29]]

Podemos analisar o movimento latitudinal das órbitas de acordo com os possíveis valores assumidos pelo parâmetro $\eta$, i.e.:

(i) Para $\eta>0$, as raízes de $\Theta_{u}$ são

$$
u_{ \pm}^{2}=\frac{a^{2}-\Phi^{2}-\eta \pm \sqrt{D}}{2 a^{2}},
$$


sendo $D \equiv\left(a^{2}-\Phi^{2}-\eta\right)^{2}+4 a^{2} \eta$. Como $\eta>0$, temos que a seguinte relação deve ser satisfeita

$$
\sqrt{D}>\left|a^{2}-\Phi^{2}-\eta\right|
$$

Isto implica que $u_{+}^{2}>0$ e $u_{-}^{2}<0$. Como $u^{2} \geq 0$, a solução $u_{-}^{2}$ deve ser descartada. Assim, para $\eta>0$, a variável $u^{2}$ está restrita ao intervalo $|u| \in\left[0,\left|u_{+}\right|\right]$, indicando que o movimento intercepta o plano equatorial repetidamente. Os pontos em que a trajetória cruza o plano equatorial recebem o nome de nodos.

(ii) Para $\eta=0$, as raízes de $\Theta_{u}$ são

$$
u_{0}^{2}=\frac{a^{2}-\Phi^{2} \pm\left|a^{2}-\Phi^{2}\right|}{2 a^{2}}
$$

que podem ser

$$
\begin{aligned}
& u_{0}^{2}=0, \\
& u_{0}^{2}=1-\frac{\Phi^{2}}{a^{2}} .
\end{aligned}
$$

Contudo, é possível provar que para órbitas esféricas de fótons devemos ter $\Phi^{2}>a^{2}$ quando $\eta=0$. Assim, a única solução aceitável fisicamente é $u_{0}^{2}=0$, pois para $\Phi^{2}>a^{2}$ teríamos $1-\frac{\Phi^{2}}{a^{2}}<0$.

(iii) Para $\eta<0$, notamos, a partir da equação (35), que uma condição necessária para que $\Theta \geq 0$ é que a seguinte relação seja verdadeira:

$$
\Phi^{2}+\eta-a^{2}<0
$$

Veremos mais adiante que esta exigência não pode ser cumprida para órbitas esféricas de fótons.

\subsection{Movimento radial}

A equação 23 pode ser reescrita em termos dos parâmetros $\eta$ e $\Phi$ como:

$$
\begin{aligned}
\left(\frac{\rho^{2} c}{E}\right)^{2} \dot{r}^{2}=R(r)= & r^{4}+\left(a^{2}-\Phi^{2}-\eta\right) r^{2} \\
& +2 m\left[\eta+(\Phi-a)^{2}\right] r-a^{2} \eta
\end{aligned}
$$

Para determinarmos as órbitas de raio constante nessa geometria devemos exigir que $r$ não varie com o parâmetro afim $\lambda$ ao longo do movimento geodésico, isto é, $\dot{r}=0$. Além disto, $\ddot{r}=0$. Essas condições são traduzidas para a função $R(r)$ da seguinte forma:

$$
R(r)=\frac{d R(r)}{d r}=0 .
$$

Isto equivale a

$$
\begin{aligned}
& r^{4}+\mathcal{A} r^{2}+2 m \mathcal{C} r-a^{2} \eta=0 \\
& 4 r^{3}+2 \mathcal{A} r+2 m \mathcal{C}=0
\end{aligned}
$$

onde

$$
\begin{aligned}
& \mathcal{A} \equiv\left(a^{2}-\Phi^{2}-\eta\right), \\
& \mathcal{C} \equiv\left[\eta+(\Phi-a)^{2}\right] .
\end{aligned}
$$

Estas relações nos levam ao seguinte conjunto de soluções:

$$
\text { (i) } \begin{aligned}
\Phi & =\frac{r^{2}+a^{2}}{a}, \quad \eta=-\frac{r^{4}}{a^{2}} ; \\
\text { (ii) } \Phi & =\frac{m\left(r^{2}-a^{2}\right)-r \Delta}{a(r-m)}, \\
\eta & =\frac{r^{3}\left[4 a^{2} m-r(r-3 m)^{2}\right]}{a^{2}(r-m)^{2}} .
\end{aligned}
$$

Note que a classe (i) não satisfaz a equação (44) para $\eta<0$. Além disso, esta classe de soluções obedece à relação

$$
\eta+(\Phi-a)^{2}=0
$$

Para esses valores de $\eta$ e $\Phi$ notamos que a única forma de a equação (31) continuar sendo compatível com a condição $\Theta \geq 0$, é se $\Phi=a \sin ^{2} \theta$. Contudo, se isso acontece, vemos que $\Theta=0 \Rightarrow \dot{\theta}=0$, o que implica $\theta=\theta_{0}=$ constante. Como isso é inconsistente com a análise feita na subseção A, a classe (i) de soluções não pode ser usada na descrição das trajetórias de órbitas de raio constante.

É possível provar que as Eqs. 52 e 53 também não são compatíveis com a condição (44), o que significa que os valores físicos permitidos para $\eta$ são tais que $\eta \geq 0$. Os valores de $r$ que permitem órbitas com $\eta \geq 0$ estão restritos ao intervalo dado pelas raízes de

$$
4 a^{2} m-r(r-3 m)^{2}=0
$$

a saber,

$$
\begin{aligned}
& r_{1} \equiv 2 m\left\{1+\cos \left[\frac{2}{3} \arccos \left(-\frac{a}{m}\right)\right]\right\}, \\
& r_{2} \equiv 2 m\left\{1+\cos \left[\frac{2}{3} \arccos \left(\frac{a}{m}\right)\right]\right\} .
\end{aligned}
$$

Estes são os valores das órbitas circulares diretas e retrógradas 9 respectivamente, de fótons no plano equatorial do espaço-tempo de Kerr.

Tendo em mente a definição clássica de momento angular, $\vec{L}=\vec{r} \times \vec{p}$, podemos entender de maneira heurística como o arrasto de referenciais explica o fato de a órbita direta ser mais próxima do BN do que a órbita retrógrada. Uma vez que um fóton em movimento co-girante tem sua velocidade aumentada devido ao maior arrasto sofrido, para que o momento angular deste fóton em torno do eixo de simetria mantenha-se com o mesmo valor, sua trajetória deve estar mais próxima do horizonte de eventos, justamente para contrabalancear o maior momento

\footnotetext{
${ }^{9}$ Chamamos de órbitas diretas aquelas em que a partícula executa um movimento co-girante com o $\mathrm{BN}$, enquanto órbitas retrógradas são aquelas em que o sentido de rotação da partícula é contrário ao sentido de rotação do BN.
} 
linear. Seguindo este raciocínio, a órbita retrógrada é mais afastada do BN devido ao menor momento linear da partícula no movimento contra-girante.

Além disso, podemos computar as raízes de $\Phi=0$, o que nos conduz a uma única solução fisicamente aceitáve 10

$$
r_{3} \equiv m\left\{1+2 \sqrt{m^{2}-\frac{1}{3} a^{2}} \cos \left(\frac{1}{3} \arccos \mathcal{U}\right)\right\},
$$

onde

$$
\mathcal{U} \equiv \frac{m\left(m^{2}-a^{2}\right)}{\left(m^{2}-\frac{1}{3} a^{2}\right)^{3 / 2}} .
$$

Esta é a única órbita de raio constante que alcança os pólos.

A variação dos parâmetros $\Phi$ e $\eta$ calculados a partir das Eqs. 52 e (53), em função da mudança de $r$, está ilustrada na Figura 1. Para órbitas circulares diretas, $r_{1} \leq r<r_{3}$, os parâmetros $\Phi$ e $\eta$ podem assumir o mesmo valor. Por sua vez, isso nunca é possível para órbitas circulares retrógradas, $r_{3}<r \leq r_{2}$,

O ponto em que a função $\Phi$ assume valores negativos, $r>r_{3}$, representa a mudança de uma órbita co-girante com o BN para uma órbita contra-girante. Os valores para os quais $\eta=0$ correspondem às trajetórias $(56) \mathrm{e}$ (57) no plano equatorial. Salientamos que a órbita circular retrógrada de raio constante possui valores negativos de $\Phi$, e que, para órbitas diretas, temos $\Phi>0$. Isto resulta de que $\Phi$ está associado com o momento angular da partícula em relação ao eixo de simetria.

Outra maneira de se perceber a presença do arrasto de referenciais é encontrando uma relação para a variação azimutal ao longo do movimento latitudinal. Isso foi obtido para o caso de órbitas do tipo tempo na referência [43] e para geodésicas nulas na referência [44].

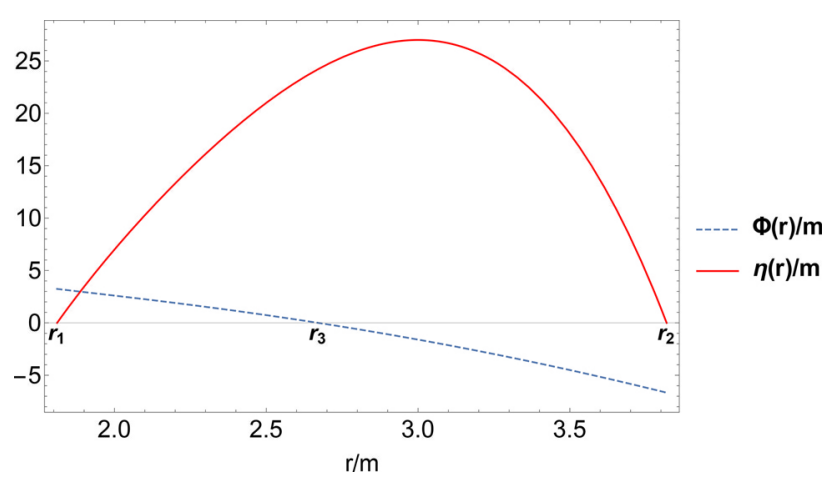

Figura 1: Comportamento dos parâmetros $\Phi / m$ e $\eta / m$ em função de $r / m$. Adotamos $a=0.8 m$ como valor do parâmetro de rotação do $\mathrm{BN}$. $r_{1}$ e $r_{2}$ são os valores do raio das órbitas luminais direta e retrógrada, respectivamente, no plano equatorial. $r_{3}$ é o raio da órbita luminal que cruza os pólos.

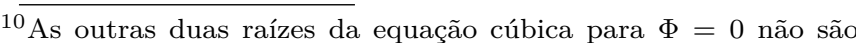
relevantes para o estudo de geodésicas, pois uma se encontra no interior do horizonte de eventos e a outra permite valores negativos de $r$.
}

Utilizando a equação 34 e definindo $z \equiv u^{2}$, obtemos, da equação 26] que:

$$
\frac{d \phi}{d z}=\frac{2 m r a-a^{2} \Phi}{2 \Delta Y(z)}+\frac{\Phi}{2 Y(z)(1-z)},
$$

onde $\Phi$ é dado pela equação 52 e

$$
Y^{2}(z) \equiv-z^{3} a^{2}-\left(\eta+\Phi^{2}-a^{2}\right) z^{2}+\eta z .
$$

Podemos reescrever $Y(z)^{2}$ como $a^{2} z\left(z_{+}-z\right)\left(z-z_{-}\right)$, onde $z_{ \pm}$são as raízes de $Y^{2}(z)$. A variação do ângulo azimutal $\phi, \Delta \phi$, após uma oscilação completa na latitude é dada por 43

$$
\Delta \phi=\frac{4 m r a-2 a^{2} \Phi}{\Delta} \int_{0}^{z_{+}} \frac{d z}{Y(z)}+2 \Phi \int_{0}^{z_{+}} \frac{d z}{Y(z)(1-z)} .
$$

A equação 62 pode ser reescrita na forma 44

$$
\Delta \phi=\frac{4}{\sqrt{z_{+}-z_{-}}}\left[\frac{(2 m r-a c \Phi) K(k)}{c \Delta}+\frac{\Phi \Pi(n, k)}{a\left(1-z_{+}\right)}\right],
$$

onde

$$
n \equiv \frac{z_{+}}{z_{+}-1} \quad \text { e } \quad k^{2} \equiv \frac{z_{+}}{z_{+}-z_{-}},
$$

com $K(n)$ e $\Pi(n, k)$ sendo as integrais elípticas de Jacobi do primeiro e terceiro tipo, respectivamente 45]. O fato de haver uma variação no ângulo azimutal no decorrer do movimento latitudinal exemplifica o arrasto dos nodos das órbitas. Isso ocorre devido ao arrasto de referenciais, efeito presente em espaços-tempos estacionários e axissimétricos, como já comentado anteriormente.

Devido a este fenômeno de arrasto dos nodos, as órbitas de raio constante com incidência fora do plano equatorial são esféricas, e não circulares. Assim, esta geometria caracteriza-se pela presença de uma fotosfera ao redor do BN de Kerr. Esclarecemos, no entanto, que a denominação esféricas resulta de as órbitas terem coordenada radial de Boyer-Lindquist constante. As superfícies $r=$ constante, nestas coordenadas, são topologicamente esféricas, mas, geometricamente, são esferoides (e não esferas).

\section{Resultados}

Nesta seção ilustramos o comportamento de algumas órbitas esféricas de partículas não massivas (OEF), exibidas nas Figuras. 2-6. Em todas estas figuras a posição inicial escolhida foi $\theta=\pi / 2$ e $\phi=0$. Nestas imagens, exibimos também as órbitas circulares de fótons, direta $\left(r_{1}\right)$ e retrógrada $\left(r_{2}\right)$, no plano equatorial.

Como podemos perceber, a órbita direta ilustrada pela Figura 2 não alcança os polos durante seu movimento latitudinal. Além disso, é possível notar (na visão frontal) que o fóton cruza o plano equatorial com $\dot{\phi} \neq 0$, o 


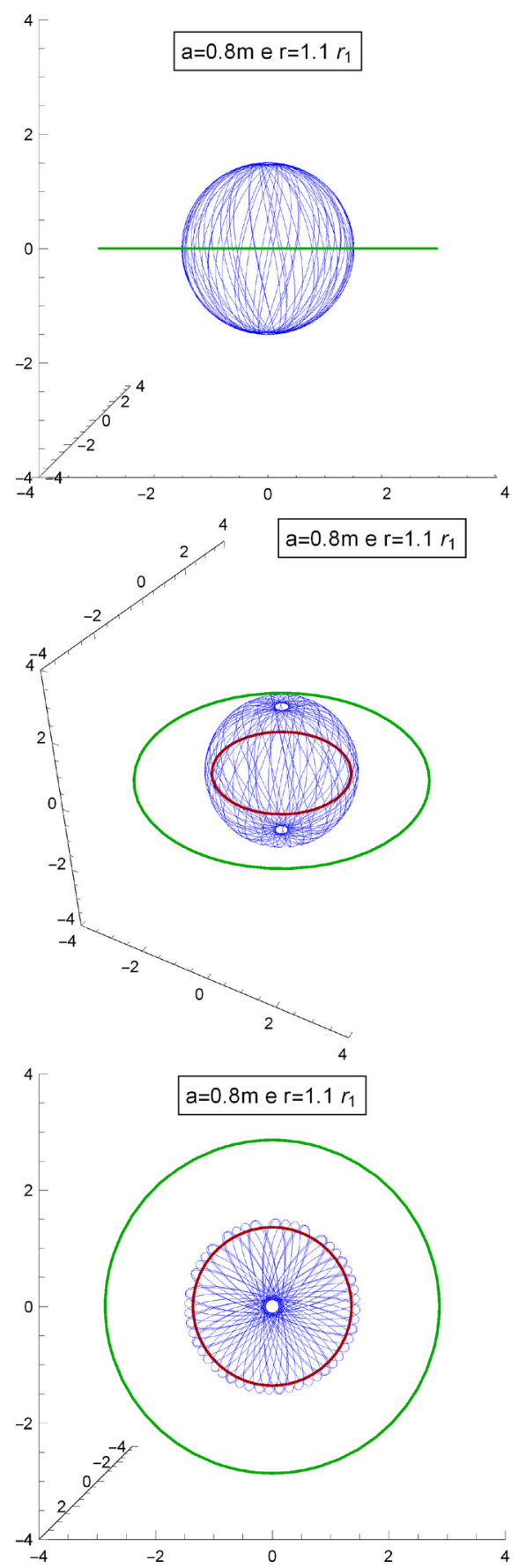

Figura 2: Visão a partir do plano equatorial (em cima), inclinada (no centro) e polar (em baixo) da órbita esférica direta de uma partícula não massiva para um BN com parâmetro de rotação $a=0.8 m$. Escolhemos o raio da órbita $r=1.1 r_{1}$. As linhas verde e vermelha representam as órbitas circulares retrógrada $\left(r_{2}\right)$ e direta $\left(r_{1}\right)$ de fótons, respectivamente, no plano equatorial.

que é justificável pela presença do efeito de arrasto de referenciais.

$\mathrm{Na}$ órbita direta representada na Figura 3, que está mais próxima de $r_{1}$ [cf. equação (56)], percebemos que o plano limite das trajetórias tende ao plano equatorial, como esperado. Ademais, o arrasto de nodos pode ser claramente notado na visão frontal, com o deslocamento
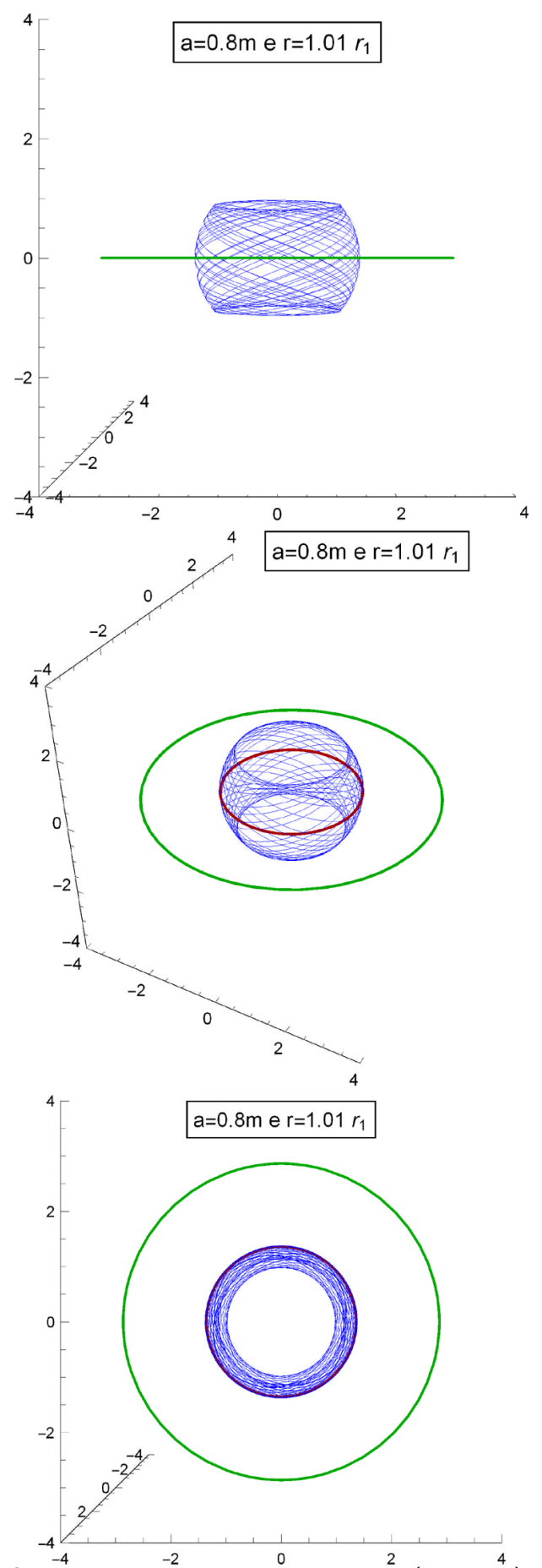

Figura 3: Visão a partir do plano equatorial (em cima), inclinada (no centro) e polar (em baixo) da órbita esférica direta de uma partícula não massiva para um BN com parâmetro de rotação $a=0.8 m$. Raio da órbita $r=1.01 r_{1}$. Assim como na Figura 2 as linhas verde e vermelha representam as órbitas circulares retrógrada $\left(r_{2}\right)$ e direta $\left(r_{1}\right)$ de fótons, respectivamente, no plano equatorial.

azimutal da trajetória ao interceptar o plano equatorial. Observamos ainda que a órbita descreve uma esfera menor do que no caso da Figura 2

A Figura 4 ilustra o comportamento da órbita esférica de raio $r=r_{3}$ [cf. Eq (58)], que representa a única órbita esférica a alcançar os polos do $\mathrm{BN}$ de Kerr. O momento 

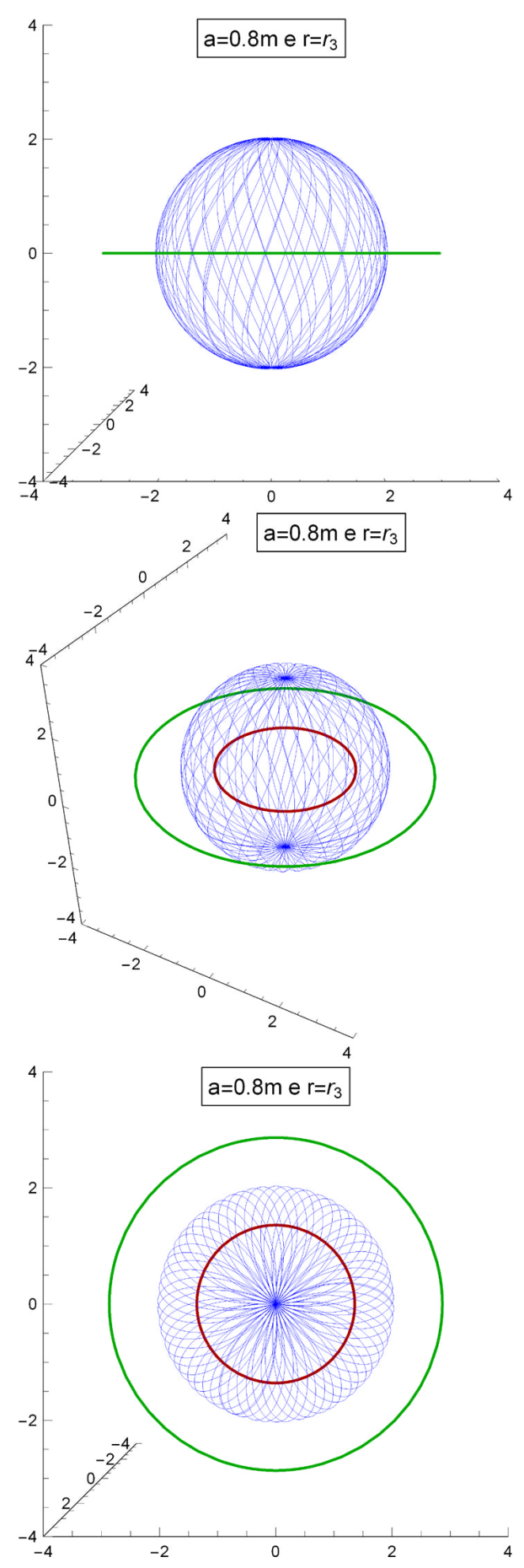

Figura 4: Visão a partir do plano equatorial (em cima), inclinada (no centro) e polar (em baixo) da órbita esférica de uma partícula não massiva para um $\mathrm{BN}$ com parâmetro de rotação $a=0.8 \mathrm{~m}$. Para esta órbita temos $r=r_{3}$, o que corresponde a uma órbita com momento angular azimutal nulo. Representamos também as órbitas circulares retrógrada $\left(r_{2}\right)$ e direta $\left(r_{1}\right)$ de fótons no plano equatorial, a exemplo das Figuras 2 e 3

angular em torno do eixo de simetria é nulo para esta órbita $(\Phi=0)$.

A Figura 5 representa a órbita esférica direta do fóton para um valor de raio tal que os parâmetros $\Phi$ e $\eta$ sejam
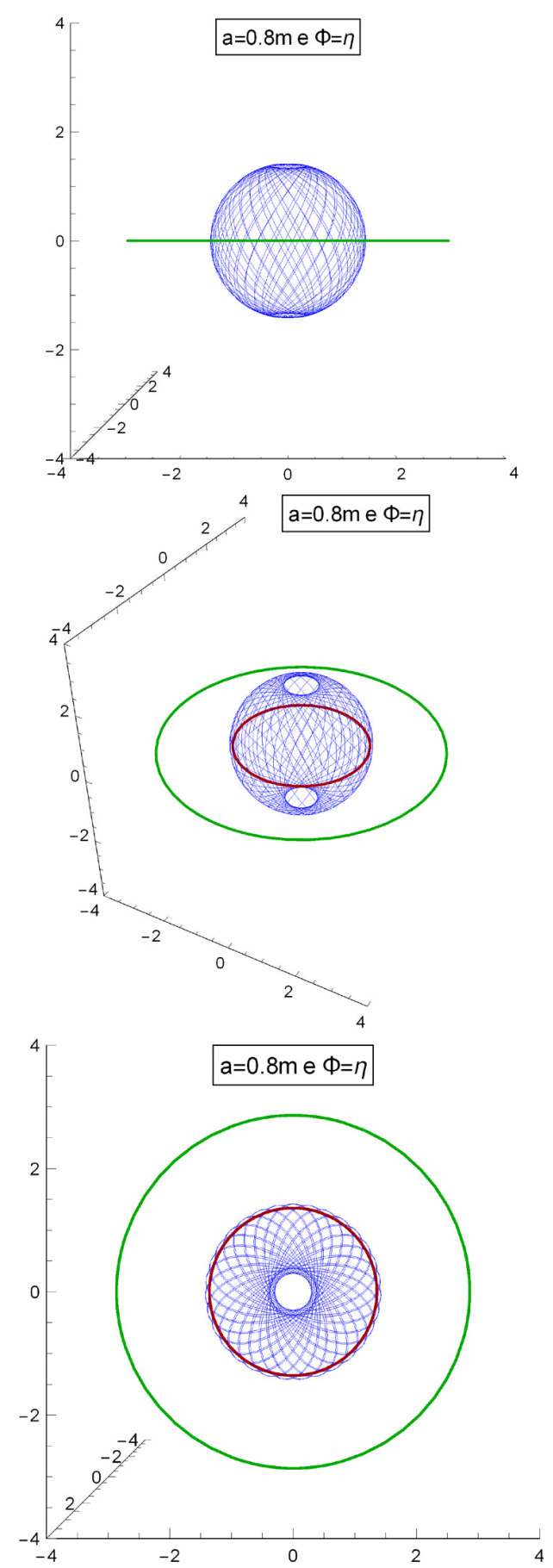

Figura 5: Visão a partir do plano equatorial (em cima), inclinada (no centro) e polar (em baixo) da órbita esférica direta de uma partícula não massiva para um BN com parâmetro de rotação $a=0.8 \mathrm{~m}$. Para esta órbita escolhemos $\Phi=\eta$. Assim como nas Figura: $23 \mathrm{e} 4$ as órbitas circulares fotônicas no plano equatorial estão representadas pelas linhas verde e vermelha.

iguais. Note-se que $\Phi=\eta$ apenas para órbitas diretas $(\Phi>0$, ver Figura 1).

A Figura 6 retrata uma órbita retrógrada de raio $r=$ $3.75 \mathrm{~m}$. Note que, neste caso, o momento angular do fóton é grande o suficiente para superar o efeito de arrasto de referenciais, realizando o movimento contra-girante. 

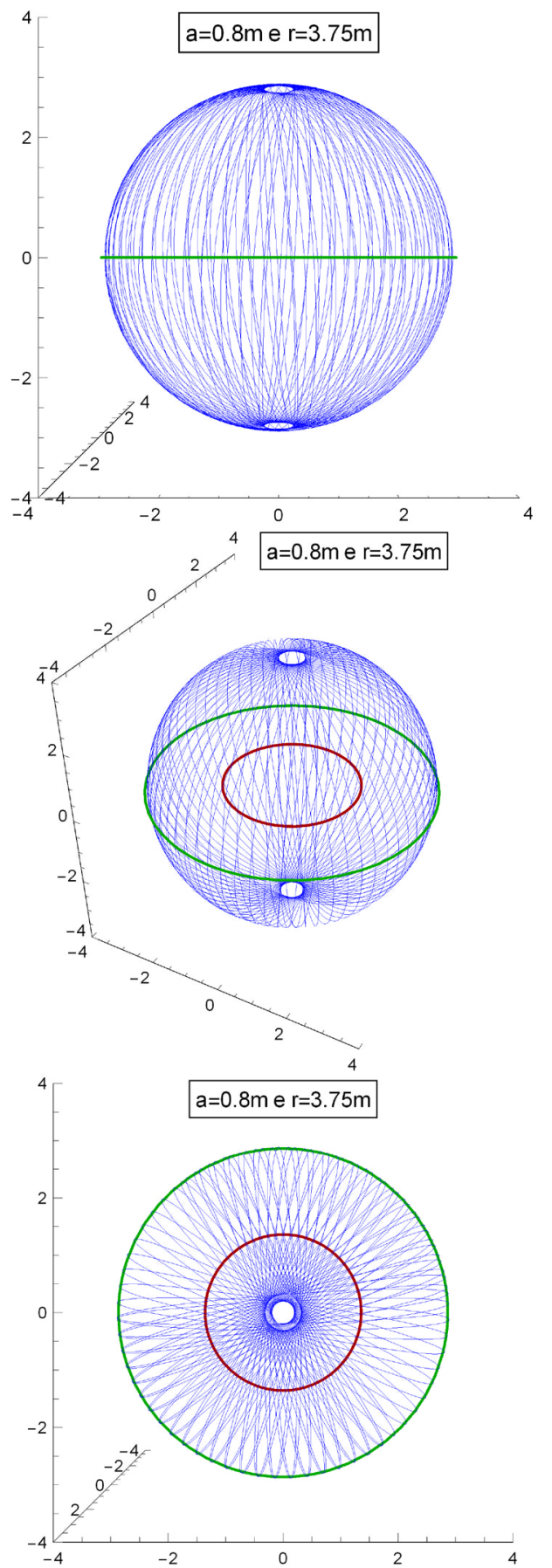

Figura 6: Visão a partir do plano equatorial (em cima), inclinada (no centro) e polar (em baixo) da órbita esférica retrógrada (com raio $r=3.75 \mathrm{~m}$ ) de uma partícula não massiva para um $\mathrm{BN}$ com parâmetro de rotação $a=0.8 \mathrm{~m}$. Exibimos também as órbitas circulares retrógrada (em verde) e direta (em vermelho) de fótons no plano equatorial, como nas Figuras $2,3,4$ e 5.

Todos as imagens desta seção ilustram OEF para um BN de Kerr com momento angular $a=0.8 m$. O aumento do valor do parâmetro de rotação do BN resulta em órbitas circulares diretas de fótons no plano equatorial mais próximas do horizonte de eventos, enquanto que as órbitas circulares retrógradas de fótons no plano equatorial ocorrem em um valor de $r$ mais distante do horizonte de eventos. Exemplos de OEF para o caso de um BN de Kerr extremo podem ser encontradas na Ref. [44].

\section{Conclusão}

Após uma breve revisão sobre o espaço-tempo de um BN de Kerr, obtivemos as equações que descrevem as geodésicas - tanto de partículas massivas quanto não massivas - nessa geometria. Para isso, utilizamos a separabilidade da equação de Hamilton-Jacobi e a definição da constante de Carter. Em seguida, fizemos uma análise dessas equações para as órbitas esféricas de fótons (OEF) e mostramos exemplos de algumas dessas órbitas. O estudo dessas órbitas é de grande importância, pois ajuda a entender a fenomenologia do campo gravitacional de um BN com rotação.

As órbitas fundamentais de fótons delimitam a região em que radiação próxima ao $\mathrm{BN}$ pode chegar até um observador no infinito, determinando assim a forma da sombra do BN. Além disso, um fóton que passe próximo de um órbita fundamental pode ser espalhado com grandes ângulos. Isto faz com que, à medida que nos aproximamos das extremidades da sombra do buraco negro, apareçam cópias da esfera celeste [21]. Contudo, essas séries de imagens são altamente desmagnificadas, o que torna difícil distinguir umas das outras 46.

Uma possível extensão deste trabalho seria calcular a sombra do BN de Kerr, uma vez conhecidas as OEF desse sistema. Isto ajudaria a visualizar o cenário que se espera encontrar nas observações astronômicas de BNs.

\section{Agradecimentos}

Agradecemos a Pedro V. P. Cunha por muitas discussões sobre este tema. Agradecemos também o apoio do Conselho Nacional de Desenvolvimento Científico e Tecnológico (CNPq), da Coordenação de Aperfeiçoamento de Pessoal de Nível Superior (CAPES) - Código de Financiamento 001, da Fundação para a Ciência e a Tecnologia (FCTPortugal) através dos projetos UID/MAT/04106/2019 (CIDMA) e PTDC/FIS-OUT/28407/2017. Este trabalho também foi apoiado pela União Europeia, através do projeto H2020-MSCA-RISE-2017 Grant No. FunFiCO777740. C. H. agradece à UFPA pela hospitalidade durante várias visitas.

\section{Referências}

[1] C.A.R. Herdeiro e J.P.S. Lemos, Gazeta de Física 41, 2 (2018).

[2] K. Schwarzschild, Sitzungsber. Preuss. Akad. Wiss. Berlin (Math. Phys.) 1916, 189 (1916).

[3] A. Saa, Rev. Bras. Ensino Fís. 38, e4201 (2016).

[4] R. Narayan e J.E. McClintock, arXiv:1312.6698 (2013).

[5] B.P. Abbott, R. Abbott, T.D. Abbott, M.R. Abernathy, F. Acernese, K. Ackley, C. Adams, T. Adams, P. Addesso, 
R.X. Adhikari et al., Phys. Rev. Lett. 116, 061102 (2016).

[6] J. Kormendy e D. Richstone, Annu. Rev. Astron. Astrophys. 33, 581 (1995).

[7] M. Visser, arXiv:0706.0622 (2007).

[8] S. Teukolsky, Class. Quantum Grav. 32, 124006 (2015).

[9] J.M. Bardeen, Nature 226, 64 (1970).

[10] R.P. Kerr, Phys. Rev. Lett. 11, 237 (1963).

[11] F.W. Dyson, A.S. Eddington e C. Davidson, Phil. Trans. R. Soc. Lond. 220, 291 (1920).

[12] L.C.B. Crispino e D.J. Kennefick, Nature Physics 15, 416 (2019).

[13] L.C.B. Crispino e S. Paolantonio, Nat. Astron. 4, 6 (2020).

[14] C.F.B. Macedo, L.C.S. Leite, E.S. Oliveira, S.R. Dolan e L.C.B. Crispino, Phys. Rev. D 88, 064033 (2013).

[15] L.C.S. Leite, S.R. Dolan e L.C.B. Crispino, Phys. Lett. B 774, 130 (2017).

[16] L.C.S. Leite, S. Dolan e L.C.B. Crispino, Phys. Rev. D 98, 024046 (2018).

[17] C.J. Goebel, Astrophys. J. 172, L95 (1972).

[18] V. Cardoso, E. Franzin e P. Pani, Phys. Rev. Lett. 116, 171101 (2016).

[19] P.V.P. Cunha, E. Berti e C.A.R. Herdeiro, Phys. Rev. Lett. 119, 251102 (2017).

[20] V. Cardoso, L.C.B. Crispino, C.F.B. Macedo, H. Okawa e P. Paoni, Phys. Rev. D 90, 044069 (2014).

[21] P.V.P. Cunha e C.A.R. Herdeiro, Gen. Relativ. Gravit. 50, 42 (2018).

[22] P.V.P. Cunha e C.A.R. Herdeiro, Gazeta da Física 39, 8 (2016).

[23] https://eventhorizontelescope.org/.

[24] K. Akiyama, A. Alberdi, W. Alef, K. Asada, R. Azulay, A.K. Baczko, D. Ball, M. Balokovic, J. Barret, D. Bintley et al., Atrophys. J. 875, L1 (2019).

[25] K. Akiyama, A. Alberdi, W. Alef, K. Asada, R. Azulay, A.K. Baczko, D. Ball, M. Balokovic, J. Barret, D. Bintley et al., Atrophys. J. 875, L2 (2019).

[26] K. Akiyama, A. Alberdi, W. Alef, K. Asada, R. Azulay, A.K. Baczko, D. Ball, M. Balokovic, J. Barret, D. Bintley et al., Atrophys. J. 875, L3 (2019).

[27] K. Akiyama, A. Alberdi, W. Alef, K. Asada, R. Azulay, A.K. Baczko, D. Ball, M. Balokovic, J. Barret, D. Bintley et al., Atrophys. J. 875, L4 (2019).

[28] K. Akiyama, A. Alberdi, W. Alef, K. Asada, R. Azulay, A.K. Baczko, D. Ball, M. Balokovic, J. Barret, D. Bintley et al., Atrophys. J. 875, L5 (2019).

[29] K. Akiyama, A. Alberdi, W. Alef, K. Asada, R. Azulay, A.K. Baczko, D. Ball, M. Balokovic, J. Barret, D. Bintley et al., Atrophys. J. 875, L6 (2019).

[30] P.V.P. Cunha, C.A.R. Herdeiro e E. Radu, Phys. Rev. D 96, 024039 (2017).

[31] R. D'Inverno, Introducing Einstein's Relativity (Oxford University Press, Oxford, 1992).

[32] M.P. Hobson, G. Efstathiou e A.N. Lasenby, General Relativity: An introduction for Physicists (Cambridge University Press, Cambridge, 2006).

[33] S. Carroll, Spacetime and Geometry - An Introduction to General Relativity (Pearson Education, New York, 2004).

[34] B. Carter, Phys. Rev. Lett. 26, 331 (1978).
[35] R.H. Boyer e R.W. Lindquist, J. Math. Phys. 8, 265 (1967).

[36] J. Hartle, Gravity: An Introduction to Einstein's General Relativity (Pearson Education, San Francisco, 2003).

[37] R. Wald, General Relativity (University of Chicago Press, Chicago, 1984).

[38] J.P.B. Brito, R.P. Bernar, C.L. Benone e L.C.B. Crispino, Rev. Bras. Ensino Fís. 42, 20200015 (2020).

[39] N.A. Lemos, Mecânica Analítica (Editora Livraria da Física, São Paulo, 2007).

[40] B. Carter, Phys. Rev. 174, 1559 (1968).

[41] S. Chandrasekhar, The Mathematical Theory of Black Holes (Oxford University Press, Oxford, 1983).

[42] B. Carter, Commun. Math. Phys. 10, 280 (1968).

[43] D.C. Wilkins, Phys. Rev. D. 5, 814 (1972).

[44] E. Teo, Gen. Rel. Grav. 35, 1909 (2003).

[45] M. Abramowitz, Handbook of Mathematical Functions, with Formulas Graphs and Mathematical Tables (Dover Publications, New York, 1974).

[46] S.E. Gralla, D.E. Holz e R.M. Wald, Phys. Rev. D. 100, 024018 (2019). 